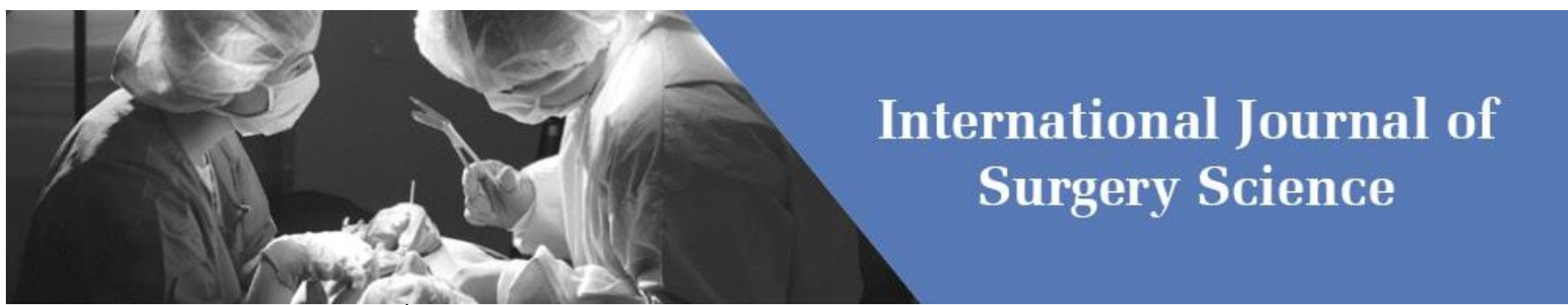

E-ISSN: 2616-3470

P-ISSN: 2616-3462

(C) Surgery Science

www.surgeryscience.com

2020; 4(1): 291-294

Received: 04-11-2019

Accepted: 08-12-2019

Luiz Eduardo Correia Miranda Department of Surgery, Oswaldo Cruz University Hospital, Recife, Pernambuco, Brazil

Américo Gusmão Amorim

Department of Surgery, Oswaldo

Cruz University Hospital, Recife,

Pernambuco, Brazil

Ana Clara Galindo Miranda Medical student, University of

Pernambuco, Recife, Brazil

Diego Laurentino Lima Research Fellow, Department of Surgery, Montefiore Medical Center, New York, United States

Raquel Nogueira Cordeiro

Research Fellow, Department of

Surgery, Montefiore Medical

Center, New York, United States
Corresponding Author: Diego Laurentino Lima Research Fellow, Department of Surgery, Montefiore Medical Center, New York, United States

\title{
Hepatic adenoma mimicking liver hydatid disease: Case report
}

\section{Luiz Eduardo Correia Miranda, Américo Gusmão Amorim, Ana Clara Galindo Miranda, Diego Laurentino Lima and Raquel Nogueira Cordeiro}

DOI: https://doi.org/10.33545/surgery.2020.v4.i1f.351

\section{Abstract}

Case Report: A 34-year-old female obese patient, married, who lived in Abreu e Lima (PE), with a history of moderate abdominal pain in the right flank and weight loss (10 kg in 7 months): She visited Para State two years ago and denied having used any oral hormonal contraceptive. Imaging exams showed big cystic and multiloculated formations in the liver, with the largest being of $16 \times 13 \times 14 \mathrm{~cm}$. Due to the aspects of the image, epidemiology and the symptoms of the patient, a diagnosis of hydatid cyst was considered, and the patient was treated with praziquantel and albendazole. The surgical procedure was proposed based on the recommendations of the WHO for the treatment of hydatic disease, even without the result of the serology test. Operatory findings showed cystic lesions with a pasty and amorphous content, of yellow green aspect. The surgical specimen evaluation showed a hepatic adenoma with necrotic tissue and central cavitation. The diagnosis was confirmed by immunohistochemistry. The serology test for echinococcosis was negative. The patient had no further complications and was discharged in good clinical condition.

Conclusion: We presented rich iconographic information of all aspects of this case and reviewed all the differential diagnoses for cystic lesions of the liver.

Keywords: Hepatic adenoma, hydatid disease

\section{Introduction}

Hepatic adenomas are benign lesions, constituted of clonal proliferations of mature hepatocytes. Microscopically, they show minimum or no architectural or cytologic atypia. In many cases, the liver is histologically normal, but steatosis or steatohepatitis can be found and are recognized as risk factors to inflammatory adenomas ${ }^{[1]}$

Complications arise when adenomas grow more than their blood vessels and undergo rupture, hemorrhage, thrombosis, infarction, or malign transformation. A rare complication described in the literature is the cystic degeneration of the adenomas ${ }^{[2]}$.

According to the World Health Organization (WHO), echinococcosis is endemic to South America, Eastern Europe, Russia, the Middle East, and China. In South America, the prevalence varies from 20 to $95 \%$. The most common intermediate hosts are rams, sheep, horses, and cattle. The incidence of surgical cases reflects only a fraction of the number of infected hosts, which is also only a fraction of the current prevalence in the endemic area ${ }^{[3]}$

In countries where hydatid cysts are endemic, the disease can be mistaken for other cystic diseases, such as simple hepatic cysts or intra-hepatic biliary cystadenoma ${ }^{[4]}$. However, there are no reports in the literature regarding hepatic adenomas mimicking hydatid cysts.

\section{Case report}

A 34-year-old female obese patient, with a history of moderate pain in the right flank and loss of $10 \mathrm{kgs}$ in the last 7 months: She reported travelling to the state of Para (endemic for echinococcosis) two years ago and denied having used any oral hormonal contraceptive. Imaging exams showed voluminous, multiloculated, cystic formations in the liver, the largest measuring 16x13x14cm (Figure 1). Considering the features of the image, epidemiology and clinical aspects, a diagnosis of hydatic cyst was considered.

The patient was started on treatment with praziquantel and albendazole, and the surgical team indicated the surgical procedure based on the WHO guidelines for the treatment of hydatic cyst pending serology. Table 1 shows her pre-operative blood tests 
Table 1: Pre-operative blood tests

\begin{tabular}{|c|c|}
\hline Blood tests & Patient (normal range) \\
\hline Bilirubin (total/D/I) & $0.3 / 0.1 / 0.2 \mathrm{mg} / \mathrm{dL}(>1.0 / 0.3 / 0.7)$ \\
\hline Alkaline phosphatase & $651 \mathrm{U} / \mathrm{L}(65-300 \mathrm{U} / \mathrm{L})$ \\
\hline GGT & $729.9 \mathrm{U} / \mathrm{L}(7$ a $32 \mathrm{U} / \mathrm{L})$ \\
\hline AST/ALT & $103.1 / 160.7 \mathrm{U} / \mathrm{L}$ (menor que 32/31 U/L) \\
\hline Albumin & $3.98 \mathrm{~g} / \mathrm{dL}(3.3 \mathrm{a} 5.2 \mathrm{~g} / \mathrm{dL})$ \\
\hline Prothrombin time $/ \mathrm{RNI}$ & $78 \%$ of normal $/ 1.18$ \\
\hline $\mathrm{HB} / \mathrm{Ht}$ & $13,5 \mathrm{~g} / \mathrm{dL} / 42 \%$ \\
\hline Serology for HBV and HCV & Negative \\
\hline $\begin{array}{c}\text { CA-125/CA19-9/CEA e } \\
\text { alpha } 1 \text { fetoprotein }\end{array}$ & Normal values \\
\hline
\end{tabular}

Intraoperatory findings showed lesions, apparently cystic, and with yellow-green, amorphous, pasty content. The study of the surgical specimen showed a hepatic adenoma with central cavitation and necrosis, which was confirmed by immunohistochemistry. (Figure 2)

The serology for echinococcosis was negative. The patient had a satisfactory post-operatory period with no complications and was discharged in good clinical condition.

\section{Discussion}

Hepatocellular adenoma is a rare benign neoplasia. It is subdivided into four types according to the Bordeaux group by classification of the genotype-phenotype: inflammatory, nuclear alpha-1 mutagenic factor, beta-catenin mutated, or with no classification $^{[5,6]}$

This classification is clinically important for the decision as to the treatment, due to a higher possibility of malignant transformation in determined subtypes. MRI is considered an effective diagnostic imaging method to distinguish the different subtypes of hepatocellular adenoma ${ }^{[7]}$.

The main question to be considered in this case is the clinical presentation of the mass. Hepatocellular adenomas are solid masses, well delimited, and heterogenous in the ultrasound. Anechoic areas correspond to focal hemorrhage or necrosis ${ }^{[8]}$. In the CAT scan, they are predominantly isodense, but they can be hypodense or heterogenous, mainly in lesions bigger than $4 \mathrm{~cm}$ in diameter. After the injection of venous contrast, these lesions are mainly hypervascular and heterogenous in the arterial phase and become isodense or hypodense in the portal phase ${ }^{[9]}$. MRI is considered the best diagnostic imaging test for a Hepatocellular adenoma due to its high sensitivity to fat and hemorrhage. The lesion presents as hyperintense or isointense in the T-1 phase, and mildly hyperintense in the T-2 phase. The presence of fat can be evaluated in images with fat suppression. There are variable characteristics in relation to the vascularization or the dynamics after the injection of gadolinium, but in most cases it presents as hypervascular in the arterial phase. Heterogeneity in the signal intensity is one of the most constant characteristics of the adenoma and results from the high variation of pathologic changes that can show in the tumor ${ }^{[5,10]}$.

In rare cases, an adenoma can present as a $17 \mathrm{~cm}$ mass with central cavitation secondary to necrosis to the point to mimicking a cystic lesion in the imaging examinations.

Rare neoplasias of the liver can present as a cyst and should be included in the differential diagnosis: cystic mucinous neoplasia of the liver and intraductal papillary neoplasia of the biliary tree are rare cystic and multiloculated neoplasias of the liver, which can reach a diameter as big as $28 \mathrm{~cm}$ and can be easily mistaken for hydatid disease in endemic areas ${ }^{[4,11]}$.

Other lesions present as cysts, but are hardly diagnosed as hydatid cysts. Ciliated hepatic cyst derived from intestines is a rare embryonic lesion and can present malign degeneration ${ }^{[12]}$.

Mesenchymal hamartoma of the liver can present as a big cystic lesion and demands a liver transplantation ${ }^{[13]}$. Endometriosis is rarely described in the liver, but can be easily mistaken for other hepatic tumors, and can present clinically as a giant cyst of the liver ${ }^{[14]}$.

The presence of a hydatid cyst occurs due to infection by $E$. granulosus. Humans get infected by eating eggs of the larvae in contaminated food. In the imaging exams, the hydatid cyst presents as a uni-or multilocular cyst, partially or completely calcified, depending on the state of infection by the parasite. When unilocular, the cyst can be similar to a simple hepatic cyst [15].

Our patient did not take any hormonal contraceptives and the MRI showed expansive cystic formations with septs and thick walls, with satellite lesions. The radiology team suggested the diagnosis of hydatid cyst due to the characteristics of the lesion in the MRI, associated with the clinical aspects and epidemiology of the patient. Analysis of the surgical specimen demonstrated that there were no true cysts, but cavities in the tumor caused by necrosis of the adenoma. The satellite cysts identified in the current case were only secondary cavities in a solitary tumor. The apparent image of the secondary cysts led to the mistaken suggestion of hydatid disease of the liver.

Hepatocellular adenomas are asymptomatic lesions, almost always diagnosed incidentally in imaging exams. The main complications are hemorrhage and malignant degeneration. Hemorrhage occurs in up to $25 \%$ of the cases and is due to the high vascularization of the tumor. The patient may be referred with unspecific symptoms such as the discomfort of abdominal pain, anemia, or present acutely ill, with shock due to intraperitoneal bleeding. Apparently, in the current case an atypically large hepatocellular adenoma evolved with limited intra-tumoral hemorrhage and central necrosis, with subsequent cavitation of the mass.

In this case, the surgical resection of the mass was indicated even with no definitive confirmation of the diagnosis. Even though it was not a hydatid cyst, there was surgical indication due to the symptoms of the patient.

In conclusion, in rare cases a hepatocellular adenoma of the liver can present as a large hepatic mass with central necrosis, suggesting a cystic lesion, and should, therefore, be considered as a differential diagnosis of cystic lesions of the liver. This form of clinical presentation is atypical. There is no similar case described in the medical literature.

\section{Subtitles}




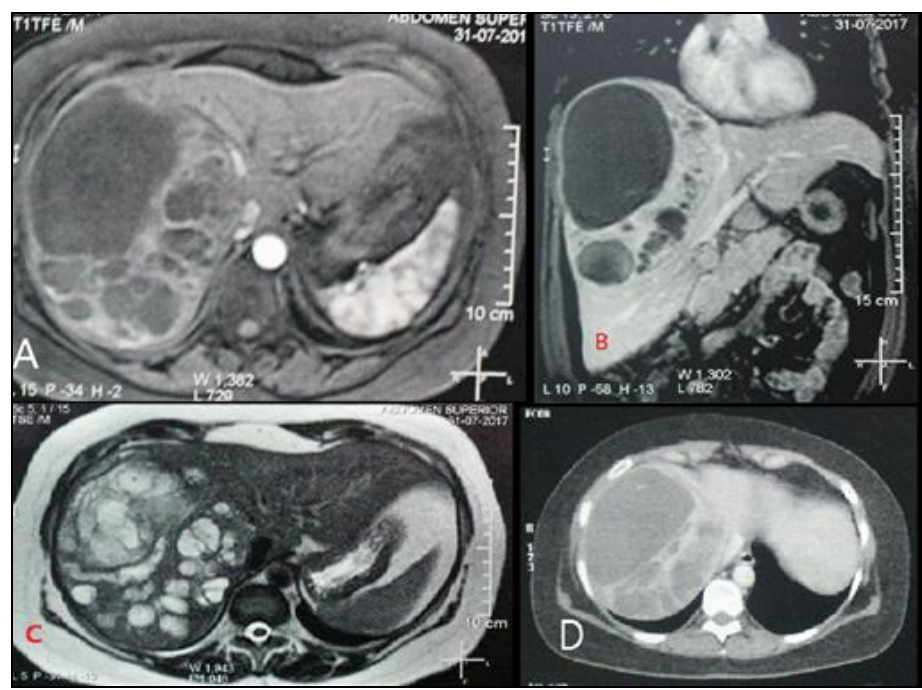

Fig 1: A-C: Expansive cystic lesions with septation, thick walss, and multiple satellite lesions (echinococcosis; D CAT Scan - axial abdomen: voluminous cystic formation in the right hepatic lobe.

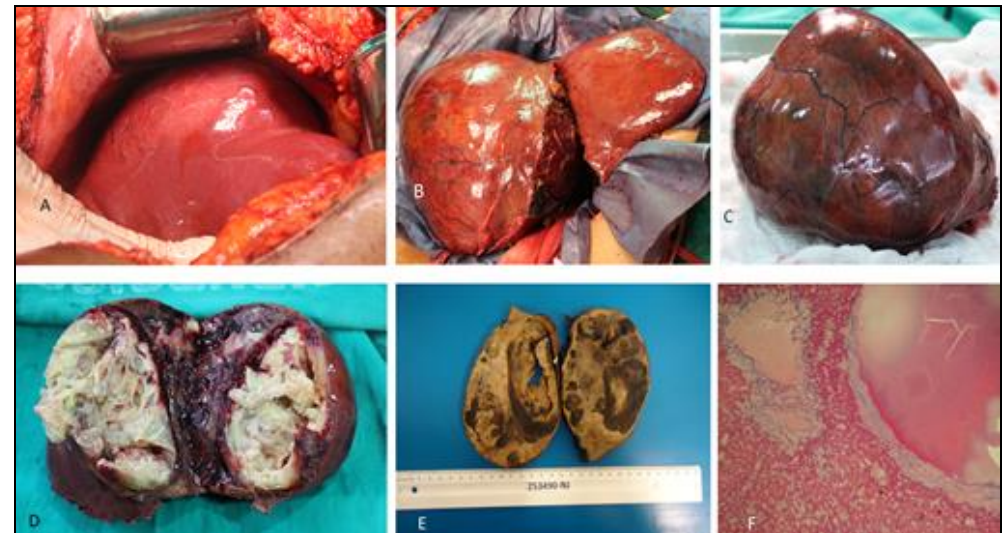

Fig 2: Nodular lesion in the right hepatic lobe with $15 \mathrm{~cm}$ in its biggest diameter, presenting solid areas and cavities filled with amorphous and thematic material. Microscopy: solid areas with proliferation of mature hepatocytes distributed in multicellular trabecula, showing sinusoid dilatation, necrosis and recent hemorrhage. A: surgical aspect; B: transoperatory period; C: lesion; D: surface of the specimen; E: Specimen in formalin; F: microscopy $200 \mathrm{x}$.

\section{References}

1. Torbenson M. Hepatic adenomas: Classification, controversies, and consensus. Surg Pathol Clin. 2018; 11(2):351-366. Doi: 10.1016/j.path.2018.02.007.

2. Foo SY, Paul L, Viswanathan S. Cystic degeneration of hepatic adenoma: A rare complication of hepatic adenoma. BJR Case Rep. 2017; 4:20170056.

3. Bhutani N, Kajal P. Hepatic echinococcosis: A review. Ann Med Surg (Lond). 2018; 36:99-105. Doi: 10.1016/j.amsu.2018.10.032.

4. Ahmad Z, Uddin N, Memon W, Abdul-Ghafar J, Ahmed A. Intrahepatic biliary cystadenoma mimicking hydatid cyst of liver: A clinicopathologic study of six cases. J Med Case Rep. 2017; 11(1):1-7. Doi:10.1186/s13256-017-1481-2.

5. Wang $\mathrm{H}$, Yang $\mathrm{C}$, Rao $\mathrm{S}$ et al. MR imaging of hepatocellular adenomas on genotype-phenotype classification: A report from China. Eur J Radiol. 2018; 100(180):135-141. Doi:10.1016/j.ejrad.2018.01.023.

6. Bioulac-Sage P, Sempoux C, Balabaud C. Hepatocellular adenomas: Morphology and genomics. Gastroenterol Clin North Am. 2017; 46(2):253-272. Doi:10.1016/j.gtc.2017.01.003.

7. Grazioli L, Olivetti L, Mazza G, Bondioni MP. MR imaging of hepatocellular adenomas and differential diagnosis dilemma, Int. J Hepatol, 2013, 374170.
8. Ronot MVV. Imaging of benign hepatocellular lesions: Current concepts and recent updates. Clin Res Hepatol Gastroenterol. 2014; 38(6):61-688. Doi:10.1016/j.clinre.2014.01.014.

9. Van Den Esschert JW, Van Gulik TM, Phoa SSKS. Imaging modalities for focal nodular hyperplasia and hepatocellular adenoma. Dig Surg. 2010; 27(1):46-55. Doi:10.1159/000268407.

10. Dharmana H, Saravana-Bawan S, Girgis S, Low G Hepatocellular adenoma: Imaging review of the various molecular subtypes. Clin Radiol. 2017; 72(4):276-285. Doi:10.1016/j.crad.2016.12.020.

11. Takano Y, Nagahama M, Yamamura E et al. Prolapse into the bile duct and expansive growth is characteristic behavior of mucinous cystic neoplasm of the liver: Report of two cases and review of the literature. Clin J Gastroenterol 2015; 8(3):148-155. Doi:10.1007/s12328-015-0569-8.

12. Bishop KC, Perrinocm, Ruzinova MB, Brunt EM. Ciliated hepatic foregut cyst : A report of 6 cases and a review of the English literature. Diagn Pathol, 2015, 2-7. Doi:10.1186/s13000-015-0321-1.

13. Li J, Cai JZ, Guo QJ et al. Liver transplantation for a giant mesenchymal hamartoma of the liver in an adult: Case report and review of the literature. World J Gastroenterol. 2015; 21(20):6409-6416. Doi:10.3748/wjg.v21.i20.6409. 
14. De Riggi MA, Fusco F, Marino G, Izzo A. Giant endometrial cyst of the liver: A case report and review of the literature. G di Chir. 2016; 37(2):79-83. Doi:10.11138/gchir/2016.37.2.079.

15. Wu CH, Chiu NC, Yeh YC, Kuo Y, Yu SS, Weng CY, Chiou YY. Uncommon liver tumors Case report and literature review. Medicine (United States), 2016, 95(39). 